\title{
Explorando la ofensividad percibida de la comunicación violenta en Internet: un estudio piloto descriptivo ${ }^{1}$
}

\author{
Exploring the perceived offensiveness of violent communication on the Internet: a descriptive pilot study
}

Francisco J. Castro Toledo 2

Rebeca Bautista Ortuño

Centro CRIMIN A para el estudio y prevención de la delincuencia (Universidad Miguel Hernández de Elche)

\begin{abstract}
Resumen
Con el objetivo de evaluar la percepción de ofensividad de contenidos comunicativos online violentos y de odio en Facebook, se desarrolló un estudio piloto en el que los participantes valoraron 14 mensajes (que fueron clasificados previamente como incitación/amenaza directa a la violencia física; enaltecimiento a la violencia física; ataques contra el honor o la dignidad; incitación a la discriminación; y ofensas a la sensibilidad colectiva) de acuerdo con su carácter ofensivo percibido. Los resultados muestran una alta variabilidad entre los participantes, y cuyas evaluaciones fueron más coherentes en los mensajes que fueron clasificados como menos grave y más grave, respectivamente. Estos resultados son del máximo interés en la discusión legal actual sobre la criminalización de contenidos comunicativos online violentos y de odio debido a su potencial ofensivo. Concluimos con algunas propuestas para orientar futuras investigaciones en esta área.
\end{abstract}

Palabras claves: Discurso del odio; comunicación violenta; redes sociales; Facebook; ofensas.

\begin{abstract}
With the aim of assessing the perceived offensiveness in online violent communication and hate speech on Facebook, a pilot study was conducted in which participants rated 14 online messages (that were previously classified as incitement/direct threat of physical violence; exaltation of physical violence; offenses against honor or dignity; and offenses to collective sensitivity) according to their perceived offensiveness. Results show a high variability across participants, whose ratings were most coherent in the messages that were classified as least serious, and most serious respectively. These results are of the utmost interest in the current legal discussion on the criminalization of online violent content and hate speech given its offensive potential. We conclude with some proposals to guide future research in this area.
\end{abstract}

Keywords: Hate speech; violent communication; social networks; Facebook; offences

1 This study has received funding from the European Union's Horizon 2020 research and innovation programme under grant agreement No 740773 .

2 Autor de correspondencia: fj.castro@crimina.es 


\section{INTRODUCCIÓN}

\subsection{Redes sociales online y comunicación violenta}

La implementación mundial de las Tecnologías de la Información y la Comunicación (TIC) ha supuesto la popularización masiva del uso de las redes sociales como nuevo espacio de comunicación interpersonal (Boyd \& Ellison, 2007; Rodríguez-Ruibal \& SantamaríaCristino, 2012). Como ejemplo de ello, Twitter se sitúa alrededor de los 326 millones de usuarios activos mensuales y Facebook supera ya los 2200 millones de usuarios, según datos oficiales de ambas empresas en 2019. No cabe duda que nos encontramos ante un escenario en el que redefinimos nuestra forma de interactuar socialmente y cuyo propósito, entre otros, es el de incrementar nuestro capital social frente a una inmensa comunidad digital de la que queremos formar parte (Castell, 2009; Ellison, Steinfield \& Lampe, 2007; Soto, 2009), al mismo tiempo que se comparten intereses, actividades, experiencia, etc. (Dueñas, Pontón, Belzunegui \& Pastor, 2016).

Sin embargo, las redes sociales en particular e Internet en su conjunto han venido albergando a lo largo de su reciente y breve historia, en una clara continuación de la tradición comunicativa del espacio físico, contenidos lingüísticos y extralingüísticos (entre ellos, de naturaleza audiovisual) que podríamos enmarcar dentro de lo que se ha denominado «comunicación violenta y de odio». Por un lado, los contenidos de odio, o tradicional hate speech, apuntan, sobre todo, a todas aquellas formas de expresión, a veces siguiendo el modelo de la vieja propaganda, dirigidas contra grupos definidos por su raza, religión, orientación sexual, discapacidad, etnia, nacionalidad, edad, género, grupo social, afiliación política, o por otras características personales, funcionales o sociales, o simplemente contra quienes no comparten una ideología (Miró-Llinares, 2016). Por otro lado, existen otro tipo de contenidos comunicativos online que han sucitado, y cada vez más, el interés de los investigadores. Hablamos de un tipo de comunicación violenta que, más allá de focalizarse en la difamación y discriminación de determinados colectivos, son «words that wound» (Lawrence et al., 1993), en otros términos, palabras que ofenden e impactan considerablemente en la opinión pública y que han puesto en funcionamiento la maquinaria penal en varias ocasiones.

En España ya han sido varios los casos de comunicación violenta y de odio que han dado lugar a famosos procesos judiciales. Entre ellos podemos hacer mención a aquellos tweets de humor negro que le costaron la concejalía de cultura a Guillermo Zapata a los pocos meses de acceder al gobierno del ayuntamiento de Madrid, la multa de $6000 €$ a la que fue condenada Marisol Moreno, concejala de Alicante, por injuriar a la Corona en Twitter, el caso del cantante de Def con Dos, a quien fiscalía llegó ha solicitar hasta 20 meses de prisión por un presunto delito de enaltecimiento del terrorismo por varios tweets que publicó entre 2013 y 2014, o la condena de 3 años y medio de prisión al rapero Vatonyc por injurias a la Corona y elogiar a ETA. En otras palabras, estamos siendo testigos sobre cómo algunas de estas formas de comunicación online generan alarma social y cierta demanda de intervención jurídica. No obstane, debemos ser prudentes ya que, aunque las 
redes sociales parezcan preñadas de contenidos que pudieran ser ofensivos o de odio (Djric et al, 2015), lo que podría resultar en una sobredimensionalización del fenómeno (MiróLlinares, 2016), no parece claro que estos contenidos correspondan a una única modalidad de comunicación violenta o que todas las personas, ya sean legos o expertos en comunicación, se ofendan del mismo modo por los mismos contenidos comunicativos (cfr., Camps, 2007; Kalinsky, 2004, entre otros).

Para ambas cuestiones, la respuesta empieza por la observación del fenómeno de la comunicación en las redes sociales online, lo que hará necesaria, en una primera fase, una aproximación taxonómica a las diferentes modalidades de comunicación violenta y de odio que apuntale las bases para un correcto análisis de las palabras que dañan y ofenden en el ciberespacio.

\subsection{Análisis del discurso a través de la taxonomía de la comunicación violenta y el discurso del odio en Internet}

Intentar clasificar las diferentes modalidades de comunicación violenta no es algo novedoso entre los investigadores. Aun no siendo este el lugar más apropiado para elaborar una reconstrucción histórica con la suficiente profundidad, es importante subrayar que existen varias propuestas de taxonomía que han utilizado criterios de diferente naturaleza para alcanzar este objetivo (cfr., Jack, William \& Adler, 2015; Matsuda, 1993; MacDevitt, Levin \& Bennet, 2002; PROXI, 2015; Sobkowic \& Sobkowic 2010, entre otros). Sin embargo, el foco de atención de todas estas taxonomías sigue siendo únicamente el análisis del hate speech, esto es, del tradicional discurso del odio, obviando el hecho que apuntábamos en el apartado anterior sobre que la comunicación violenta va más allá de la mera discriminación de colectivos y engloba todos aquellos actos de habla que puedan conceptuarse como violentos. Por ello, para este estudio piloto se utilizará la taxonomía de la comunicación violenta y del odio en Internet elaborada y ampliamente justificada por Miró-Llinares (2016), la cual observa esta diferenciación. Se trata, grosso modo, de una categorización que clasifica la comunicación violenta y de odio en dos grandes grupos: a) aquellos discursos referidos a la causación de un daño físico y b) aquellos discursos que ofenden o causan un daño moral, ya sea a individuos, grupos sociales concretos o a toda la colectividad. Como se observa, esta propuesta de clasificación se construye sobre el concepto de «violencia», introduciendo una dimensión valorativa que nos permite, además de clasificar el discurso, evaluar estas conductas comunicativas por sus consecuencias, concretadas en cinco grandes categorías: $\alpha$ ) incitación/amenaza directa a la violencia física; $\beta$ ) enaltecimiento a la violencia física; $\gamma$ ) ataques contra el honor o la dignidad; $\delta$ ) incitación a la discriminación; y $\varepsilon$ ) ofensas a la sensibilidad colectiva. Estas cinco macrocategorías recogerán todas las diferentes modalidades de comunicación violenta y de odio, esto es, las unidades comunicativas mínimas violentas y de odio que podemos hallar no sólo en las redes sociales online, sino en cualquier otra actividad comunicativa humana. 
Presentada la taxonomía, el objetivo principal de este estudio piloto es establecer un grado subjetivo de ofensividad de las cinco macrocategorías propuestas por Miró-Llinares (2016) a partir de la evidencia empírica recogida en una muestra de población lega en comunicación violenta. Dicho en forma de interrogante, ¿nos ofendemos del mismo modo por los mismos contenidos comunicativos violentos?

\section{METODOS Y MATERIALES}

\subsection{Muestra}

La muestra de participantes del estudio piloto estuvo formada por 60 usuarios de Facebook residentes en España, de edades comprendidas entre los 15 y los 60 años $(M=27,6$; $D T=12,3)$, de los cuales el 38,3\% eran hombres y el $61,7 \%$ mujeres, el 75,8\% son solteros y el $77,7 \%$ tienen estudios universitarios o superiores.

\subsection{Instrumento}

Se diseñó un cuestionario ad hoc compuesto por 14 mensajes reales publicados en la red social Facebook pertenecientes a las macrocategorías de Miró-Llinares (2016): $\alpha$ ) Incitación/amenaza directa a la violencia física; $\beta$ ) Enaltecimiento a la violencia física; $\gamma$ ) Ataques al honor/dignidad; $\delta$ ) Incitación a la discriminación y $\varepsilon$ ) Ofensas a la sensibilidad colectiva. Para la elaboración del instrumento se utilizo el sistema de encuestas gratuito de Google y para la selección de los diferentes mensajes que se muestran a continuación se contó con el asesoramiento de varios de los investigadores especialistas en comunicación violenta online del centro CRÍMINA para el estudio y prevención de la delincuencia de la UMH. Los mensajes por categorías fueron los incluidos en la Tabla 1. 
Tabla 1

Mensajes publicados en Facebook y elegidos para su evaluación por los participantes

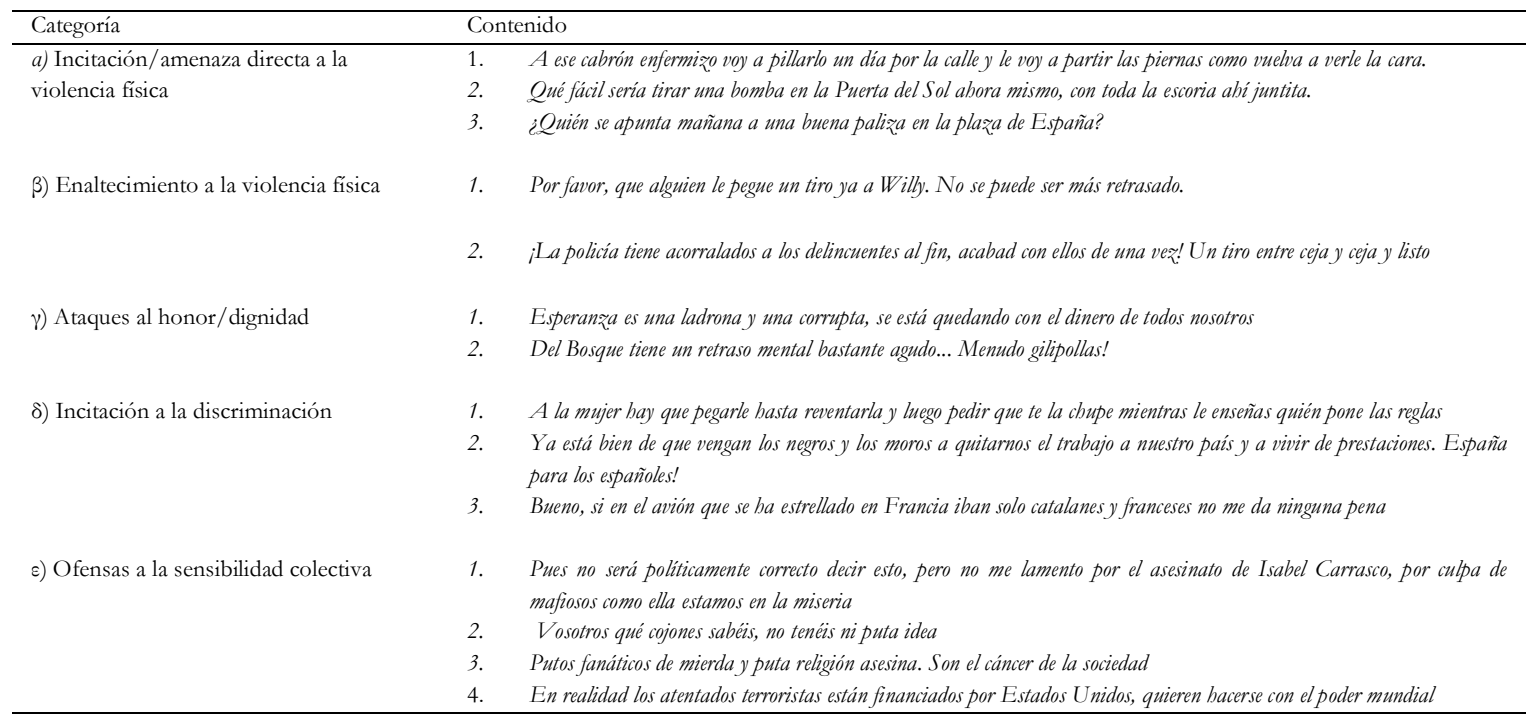

\subsection{Procedimiento}

Para la selección de los participantes y la recogida de los datos se utilizó un método no probabilístico de muestreo. Se contrataron los servicios de publicidad de Facebook para que lanzara el cuestionario durante un periodo de 5 días. Los criterios de elegibilidad para participar en el estudio fueron: 1) ser usuario de Facebook y vivir en España; 2) tener al menos 13 años de edad, y 3) hablar español. A pesar que la campaña de publicidad, únicamente se obtuvieron 60 respuestas válidas, por lo que sospechamos que fue debido a la dificultad de la tarea. En ella los participantes tenían que ordenar los mensajes presentados según cómo de ofensivos los percibían: desde el primero o más ofensivo, hasta el número 14 o menos ofensivo. Posteriormente, los datos recogidos se fueron almacenando automáticamente en un archivo de Excel y, una vez completada la base de datos con los 60 sujetos, se exportaron al paquete estadístico SPSS v. 24 para proceder a su depuración y análisis cuantitativo. 


\section{RESULTADOS}

Lo primero que se observa tras el análisis descriptivo de los datos recogidos es que solamente han sido clasificados en alguna posición todos los mensajes pertenecientes a las categorías de « $\beta$ ) enaltecimiento de la violencia física» y los de $« \gamma$ ) ataques al honor/dignidad». Del resto de categorías, hay un mensaje de cada una de ellas que no ha sido seleccionado por la mayoría de la muestra en ninguna posición en concreto, por lo que no aparecen en la lista. Nos referimos a los mensajes $\alpha 1, \delta 2$ y $\varepsilon 3$, haciendo que otros mensajes ocupen más de una posición. En segundo lugar, y con excepción de la primera y la última de las posiciones para los que existe mayor porcentaje de acuerdo entre los participantes (83,3\% y 36,7\% respectivamente), el resto de mensajes que aparecen clasificados lo están a pesar de tener porcentajes más bajos de acuerdo (véase Tabla 2).

Tabla 2

Clasificación de mensajes por orden de ofensividad percibida y numero de mensajes elegidos para la misma posición.

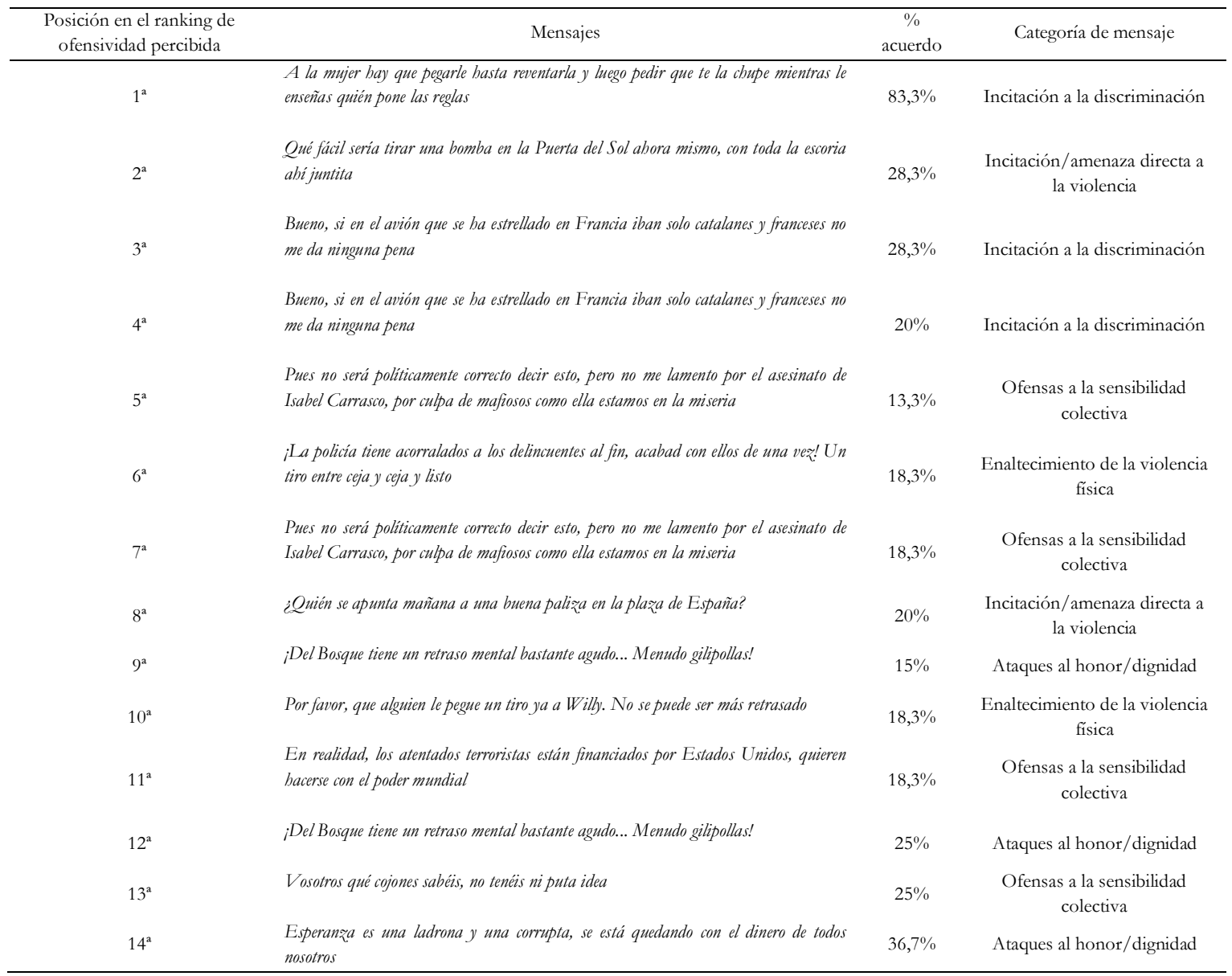

Del mismo modo, merece la pena subrayar que mientras para la primera y la última posición del ranking el número de mensajes elegidos oscila entre 5 y 6 , respecto al resto de posiciones intermedias la media de mensajes elegidos para una misma posición se eleva al doble $(M=11,25 ; D T=1,02)$. Toda esta información aparece recogida el Gráfico 1. 


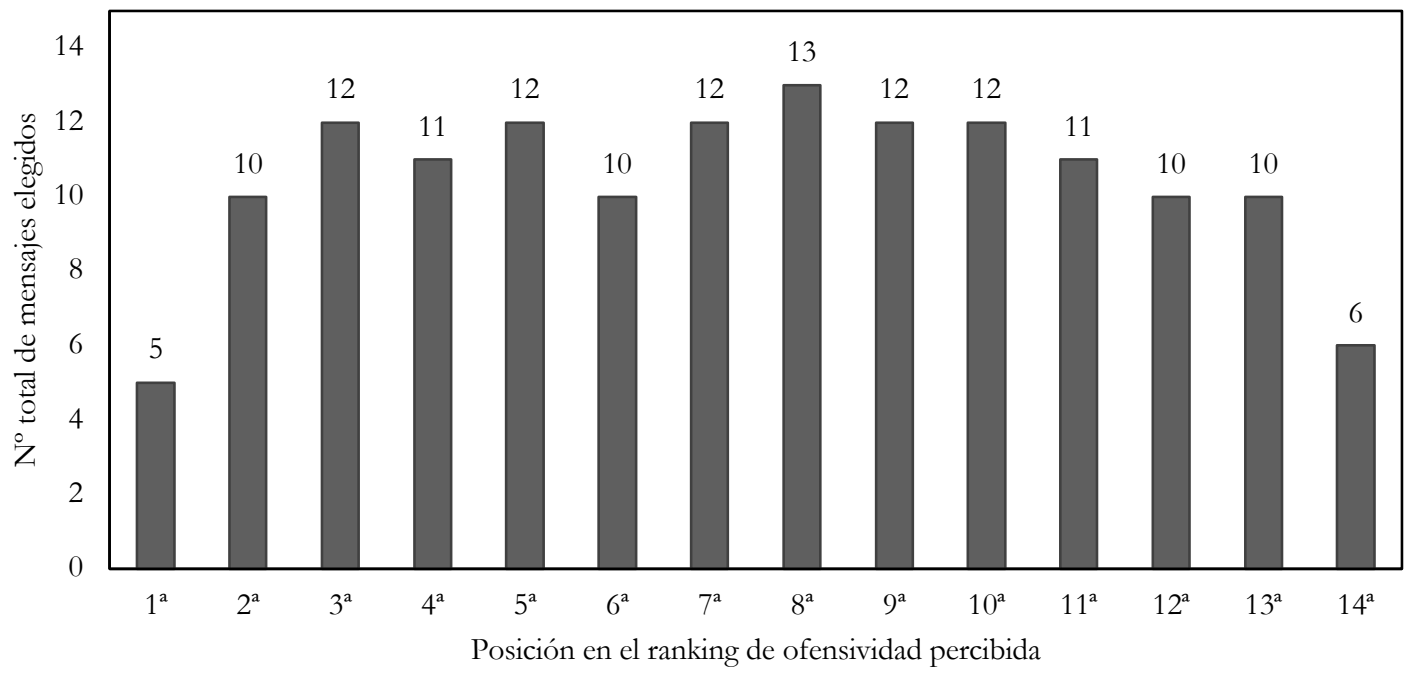

Gráfico 1. Mensajes publicados en Facebook y elegidos para su evaluación por los participantes

Sintetizando diremos, pues, que tomando los mensajes presentados como indicadores de cada macrocategoría de la taxonomía de Miró-Llinares (2016), podríamos ordenarlas por ofensividad a partir de los juicios emitidos por los participantes. La primera de ellas sería la de «incitación a la discriminación», seguida por la de «incitación/amenaza directa a la violencia». La tercera sería la categoría de «enaltecimiento de la violencia» física y en cuarto lugar la de «ofensas a la sensibilidad colectiva», ocupando esta última puestos de ofensividad media-baja. Por último, la tipología de mensajes percibidos como menos ofensivos serían los correspondientes a la categoría de «ataques al honor/dignidad».

\section{DISCUSIÓN Y CONCLUSIONES}

\section{1. ¿Relativizando la ofensa?}

De acuerdo con lo expuesto, el presente estudio piloto ha explorado el modo en el que los usuarios de la red social Facebook evalúan y clasifican la ofensividad de 14 conductas comunicativas online violentas y/o de odio. En primer lugar, los resultados que hemos obtenido muestran una lectura importante, y es que no existe, aparentemente, un patrón valorativo común de ofensividad de los mensajes publicados, excepto por 1) el gran acuerdo que el mensaje con contenido referido a violencia contra la mujer es el más ofensivo y 2) el acuerdo que el mensaje cuyo contenido acusa de corrupción a un famoso cargo público es el menos ofensivo. Con ello, se sospecha que no es la estructura formal de los mensajes, sino otras variables culturales y actitudinales de los participantes, las que podrían mediar en las evaluaciones de la ofensividad de los contenidos (cfr. Brown et al., 1986; Brown \& Williams, 1984; Costello et al., 2016; Dasgupta, 2004; Hinkle \& Brown, 1990; Walther et al., 2008). En este sentido, el acuerdo del 83,3 \% de la muestra sobre la 
mayor ofensividad de los contenidos de este tipo puede deberse, siguiendo los precedentes en la literatura, a elementos de diferentes naturaleza: entre ellos, a) el tratamiento diferencial de los medios de comunicación de la violencia machista y de género (cfr., Gámez-Fuentes y Núñez-Puente, 2013; Matterlart, 2014; Menéndez-Menéndez, 2014; Zurbano-Berenguer, 2012), b) incremento en la especialización profesional y sensibilización social frente a esta forma de delincuencia (cfr., Ferreiro-Basurto et al., 2015; Rodríguez-López y Robles Álvarez, 2016; Soleto-Muñoz, 2015;) y c) la implementación y desarrollo progresivo de políticas de igualdad (cfr., Laliga-Mollá \& Bonillas-Campos, 2015; Lombardo y León, 2015). Todo ello, en definitiva, confluye llevando esta problemática al primer nivel de repulsa social y valoración negativa, haciendo que el tratamiento y prevención de la violencia de género es una de las problemáticas sociales que recibe más cobertura política y mediática

Del mismo modo, resulta del máximo interés subrayar cómo para un 36,7 \% de los participantes del estudio, el segundo mensaje que ha aglutinado mayor acuerdo ha sido aquel considerado como el menos ofensivo y cuyo contenido hace referencia a una acusación de corrupción a un cargo político. Ciertamente, la corrupción y el fraude es el tercero de los principales problemas percibido por los ciudadanos españoles, después del desempleo y los partidos políticos en general (CIS, febrero de 2019). En este contexto, la evaluación de ofensividad de estos mensajes online podría responder, entre otros motivos, al tratamiento especialmente estigmatizante de cualquier forma de corrupción política en los medios de comunicación tradicionales y digitales (cfr., Martínez-Gallego, 2013; Palau \& Davesa, 2013; Santoveña-Casal, 2015), la percepción sobre cómo la corrupción afecta negativamente a la calidad de la democracia (cfr., Laguna-Platero, 2013; Recuero-López, 2015; Villoira y Jiménez, 2012) y, especialmente, el sentimiento de insatisfacción punitiva frente a este tipo de conductas de mala praxis pública (cfr., Mateos \& Penades, 2013; Zuñiga-Rodríguez, 2015). En suma, aunque esto debería comprobarse en investigaciones futuras, nuestros resultados nos hacen sospechar que existe cierta relación inversa entre la criminalización de un individuo o colectivo y la percepción de ofensividad de mensajes violentos o de odio dirigidos contra ellos.

\subsection{Limitaciones y prospectiva}

Debemos señalar en primer lugar que, debido a la novedad de esta taxonomía sobre comunicación violenta y de odio que se ha utilizado de referencia en este estudio, los precedentes empíricos en la literatura son aún escasos (Miró-Llinares, Moneva-Pardo y Esteve-Campello, 2018; Bautista-Ortuño, Castro-Toledo, Perea García y RodriguezGomez, 2018). Esto además hace que nuestro enfoque deba ser revisado cuidadosamente, subrayando la necesidad de apuntar algunas limitaciones a tomar en consideración en futuros estudios. Así, en primer lugar, debemos ser prudentes al considerar el alcance de los resultados derivados de una muestra de este tamaño, la cual entendemos debe ser ampliada. Asimismo, resultaría muy revelador valorar y controlar otras dimensiones que pudieran mediar en la evaluación de ofensividad de contenidos online (i.e. actitudes políticas, punitivas o cualquier otra que pudieran estar relacionadas temáticamente con el contenido 
de los mensajes), tal y como hemos comprobado en otro lugar. Además, y en virtud de conocer mejor el funcionamiento de esta nueva taxonomía, investigaciones futuras deberían de examinar la evaluación de contenidos pertenecientes a la misma macrocategoría, lo que afinaría aún más nuestros análisis.

Por otro lado, el soporte eminentemente lingüístico de los contenidos online de nuestro estudio podría naturalizarse más en un futuro combinándolo con otros contenidos de naturaleza audiovisual, esto es, otros contenidos enormemente utilizados en la comunicación en redes sociales que contribuirían a contextualizarlos de un modo más adecuado. Esto se entiende en mayor medida cuando consideramos que la comunicación violenta y de odio no sólo se construye por medio de contenidos lingüísticos, sino que el uso de contenidos audiovisuales en las redes sociales son un elemento clave en la construcción semiótica tanto de este tipo de discurso como de cualquier otro online (Castillo-Hinojosa, 2012). En esta línea, existe literatura científica focalizada en la elaboración de instrumentos para la medición de valores en contenidos audiovisuales (cfr., Rodríguez-Bravo et al., 2013) que podrían resultar complementarias con nuestro estudio.

En definitiva, los resultados de este estudio aportan información, aunque preliminar, de relevancia para conocer mejor el funcionamiento de la comunicación violenta y de odio en Internet. En especial, subraya la gran variabilidad en los juicios acerca de la valoración de la ofensividad de distintos tipos de comunicación violenta y pone de manifiesto, no solamente la complejidad del fenómeno en sí, sino además las posibles dificultades a las que se puede enfrentar el legislador en su intento de regular este tipo de comportamientos y de sancionarlos justamente. En este sentido, permite aportar estrategias empíricas prometedoras que, en combinación con las lógicas principialista y garantista propias de nuestra política legislativa penal, arrojen cierta luz al importante debate social y jurídico sobre de si deben o no ser criminalizadas estas conductas (cfr., Baker, 2007; Boeckman \& Turpin-Petrosino, 2002; Waldron, 2012; Magdy, Darwish \& Abokhoadir, 2015, Matsuda, 1993; Miró-Llinares, 2015; entre otros).

\section{REFERENCIAS}

Baker, D. J. (2007). The Moral Limits of Criminalizing Remote Harms Review. New Criminal Law Review: An International and Interdisciplinary Journal, 10(3). 370-391. doi: http://dx.doi.org/10.1525/nclr.2007.10.3.370.

Basurto-Ferreiro, V. et al. (2015). Instruments for the Analysis of Batterers' Intervention Programmes applied in Spain in Cases of Domestic Violence. Journal of Feminist, Gender and Women Studies, 2015, 2. 11-22. Disponible en: http://goo.gl/S8epSM

Bautista-Ortuño, R., Castro-Toledo, F. J., Perea-García, J. O., \& Rodríguez-Gómez, N. (2018). " May I offend you?" An experimental study on perceived offensiveness in online violent communication and hate speech. International e-Journal of Criminal Sciences, (12). 
Boeckmann, R. J. \& Turpin-Petrosino, C. (2002). Understanding the Harm of Hate Crime. Journal of Social Issues, 58(2), 207-225.doi: http://dx.doi.org/10.1111/15404560.00257

Boyd, D. M. \& Ellison, N. B. (2007). Social Network Sites: Definition, History, and Scholarship. Journal of Computer-Mediated Communication, 13, 210-230. doi:10.1111/j.1083-6101.2007. 00393.x

Brown, R., \& Williams, J. (1984). Group Identification: The Same Thing to All People?. Human Relations, 37(7), 547-564.

doi: http://dx.doi.org/10.1177/001872678403700704

Brown, R., Condor, S., Mathews, A., Wade, G., \& Williams, J. (1986). Explaining Intergroup Differentiation in an Industrial Organization. Journal of Occupational psychology, 59(4), 273-286. doi: http://dx.doi.org/10.1111/j.20448325.1986.tb00230.x

Camps, V. (2007). Ofensas y Libertad de expresión. QUA-DERNS, 27, 3-12. Disponible en: http://goo.gl/mtzbic

Castells, M.L. (2009). Comunicación y Poder. Madrid: Alianza Editorial.

Costello, M., Hawdon, J., Ratliff, T \& Grantham, T. (2016). Who Views Online Extremism? Individual Attributes Leading to Exposure. Computers in Human Behavior, 63, 311-320. doi: http://dx.doi.org/10.1016/j.chb.2016.05.033

Djuric, N., Zhou, J., Morris, R., Grbovic, M., Radosavljevic, V., \& Bhamidipati, N. (2015). Hate Speech Detection with Comment Embeddings. Proceedings of the 24th International Conference on World Wide Web Companion. http://dx.doi.org/10.1145/2740908.2742760.

Dasgupta, N. (2004). Implicit Ingroup Favoritism, Outgroup Favoritism, and their Behavioral Manifestations. Social Justice Research, 17(2), 143-169. doi: http://dx.doi.org/10.1023/B:SORE.0000027407.70241.15

Dueñas-Cid, D., Pontón-Merino, P., Belzunegui-Eraso, E, \& Pastor-Gosálbez, I. (2016). Expresiones discriminatorias, jóvenes y redes sociales: la influencia del género. Comunicar, 46(24), 67-76. doi: http://dx.doi.org/10.3916/C46-2016-07

Gámez-Fuente, M.J. \& Núñez-Puente, S. (2013). Medios, ética y violencia de género: más allá de la victimización. Asparkía. Investigació feminista, 24, 145-160. Disponible en: http://goo.gl/i91VZA

Jacks, W \& Adler, J. R. (2015). A Proposed Typology of Online Hate Crime. Psychology Unbound: Open Access Journal of Forensic Psychology, 7, 64-89. Disponible en: http://goo.gl/1hKr1l

Kalinsky, B. (2004). El contexto de la ofensa: un concepto significativo para el análisis del delito. Urbe et ius: revista de opinión jurídica, 2, 160-175. Disponible en: http://goo.gl/ZluQXP

Laliga-Mollá, M. \& Bonilla-Campos, A. (2015). Políticas públicas en el tratamiento de la violencia de género: una aproximación crítica a la eficacia de las herramientas jurídicas y alternativas. Journal of Feminist, Gender and Women Studies, 1, 41-51. Disponible en: http://goo.gl/DpXQhV 
Lawrence Iii, C. R; Matsuda, M. J; Delgado, R \& Williams Crenshaw, K. (1993). Introduction. En matsuda, m. J; lawrence iii, c. R; delgado r.; williams crenshaw, k.: Words that wound. Critical Race Theory, assaultive speech and the First Amendment. Oxford: Westview Press.

Lombardo, E., \& León, M. (2015). Políticas de igualdad de género y sociales en España: origen, desarrollo y desmantelamiento en un contexto de crisis económica. Investigaciones feministas, 5 13-35. http://dx.doi.org/10.5209/rev_INFE.2014.v5.47986

López, M. R., \& Álvarez, D. R. (2016). Institutional Advertising in Spain: Analysis of the Campaigns of the Government about Domestic Violence (2006-2015). Vivat Academia, 134, 83-104. · http://dx.doi.org/10.15178/va.2016.134.83-104.

Magdy, W., Darwish, K. Y Abokhoadir, N. (2015). Quantifying Public Response towards Islam on Twitter after Paris Attacks. arXiv preprint arXiv:1512.04570.

Manafy, M. Y Gautschi, H. (2011). Dancing with digital natives: Staying in step with the generation that's transforming the way business is done. New Jersey: Cyberage books.

Martínez-Gallego, F. A. (2013). Medios de comunicación y escándalos de corrupción en España: ¿denunciantes, magnificadores, cómplices?. OBETS: Revista de Ciencias Sociales, 8, 99-126. doi: http://dx.doi.org/10.14198/OBETS2013.8.1.04

Martínez, J. \& Manuel Goig. (2015). Transparency and Corruption. The Social Perception Before Corrupt behaviors. Revista De Derecho UNED, 17, 73-107. Disponible en: http://goo.gl/aMib2M

Mateos, A., \& Penadés, A. (2013). España: crisis y recortes. Revista de ciencia política (Santiago), 33(1), 161-183. doi: http://dx.doi.org/10.4067/S0718090X2013000100008

Matsuda, M. J. (1993). Words that wound: Critical race theory, assaultive speech, and the first amendment. Westview Press.

Mattelart, M. (2014). Género, comunicación e investigación desarrollada por mujeres. Revista de la Asociación Española de Investigación de la Comunicación, 1(2), 1-5. Disponible en: http://goo.gl/0gWtKH)

Mcdevitt, J.; Levin, J. \& Bennett, S. (2002). Hate Crime Offenders: an Expanded Typology. Journal of Social Issues, 58(2), 303-317. doi: http://dx.doi.org/10.1111/15404560.00262

Menéndez-Menéndez, M. I. (2014). Retos periodísticos ante la violencia de género: El caso de la prensa local en España. Comunicación y sociedad, 22, 53-77. Disponible en: http://goo.gl/6oA1TH

Miró-Llinares, F. (2015). La criminalización de conductas "ofensivas": A propósito del debate anglosajón sobre los «límites morales» del derecho penal. Revista electrónica de ciencia penal y criminología, 17. 1-65. Disponible en: http://goo.gl/MHbebm

Miró-Llinares, F. (2016). Taxonomía de la comunicación violenta y el odio en Internet. Revista de Internet, Derecho y Politica, 22, 82-107. Disponible en: http://goo.gl/IR79Mw

Miró-Llinares, F., Moneva, A., \& Esteve, M. (2018). Hate is in the air! But where? Introducing an algorithm to detect hate speech in digital microenvironments. Crime Science, $7(1), 15$. 
Palau, A., \& Davesa, F. (2013). El impacto de la cobertura mediática de la corrupción en la opinión pública española. Revista Española de Investigaciones Sociológicas (REIS), 144(1), 97-124. doi: http://dx.doi.org/10.5477/cis/reis.144.97

Platero, A. L. (2013). La percepción de la corrupción, factor mediatizante de la democracia. OBETS: Revista de Ciencias Sociales, 8, 79-98. doi: http://dx.doi.org/10.14198/OBETS2013.8.1.03

Prensky, M. (2001). Digital Natives, Digital Inmigrants. On the Horizon, 9 (5). Disponible en: http://goo.gl/cqbY85

Proxi (2015). Informe del «Observatorio Proyecto Online contra la Xenofobia y la Intolerancia» (Vol I). (http://goo.gl/4IYPtt) (Consulta: 4 de abril de 2017).

Recuero-López, F. (2015). La percepción de la corrupción en España y su incidencia en la desconfianza hacia las instituciones en tiempos de crisis. En J. Marco-Marco y B. Nicasio-Varea (coord.), La regeneración del sistema: reflexiones en torno a la calidad democrática, el buen gobierno y la lucha contra la corrupción (pp. 423-438). Valencia. AVAPOL

Bautista-Ortuño, R. B., Castro-Toledo, F. J., Perea-García, J. O., \& Rodríguez-Gómez, N. (2018). " May I offend you?" An experimental study on perceived offensiveness in online violent communication and hate speech. International e-journal of criminal sciences, (12), 2.

Rodriguez-Ruibal, A. Y Santamaría-Cristino, P. (2012). Análisis del uso de las redes sociales en Internet: Facebook y Twitter en las universidades españolas. Revista de comunicación y tecnologias emergentes, 10 (2), 228-246. doi: http://dx.doi.org/10.7195/ri14.v10i2.198

Sobkowicz, P.; Sobkowicz, A. (2010). Dynamics of Hate Based Internet User Networks. The European Physical Journal B, 73(4), 633-643. doi: http://dx.doi.org/10.1140/epjb/e2010-00039-0

Soto, M. (2009). Impacto de Facebook y otras redes de comunicación social en los procesos de reclutamiento y otros asuntos de recursos humanos en el ámbito laboral. Revista de estudios críticos del Derecho, 4, 113. Disponible en: http://goo.gl/YKjHLb

Villoria, M., \& Jiménez, F. (2012). La corrupción en España (2004-2010): datos, percepción y efectos. Revista Española de Investigaciones Sociológicas (REIS), 138(1), 109-134. doi: http://dx.doi.org/10.5477/cis/reis.138.109

Waldron, J. (2012). The Harm in Hate Speech. Harvard University Press.

Walther, J.B., Van-Der-Heide, B., Kim, S.Y., Westerman, D., \& Tong, S.T. (2008). The Role of Friends' Appearance and Behavior on Evaluations of Individuals on Facebook: Are We Known by the Company We Keep?. Human Communication Research, 34, 28- 49. doi: http://dx.doi.org/10.1111/j.1468-2958.2007.00312.x

Zurbano-Berenguer, B. (2012). El concepto «violencia de género» en la prensa diaria nacional española. Cuestiones de género: de la igualdad y la diferencia, 7, 25-44. http://dx.doi.org/10.18002/cg.v0i7.901

Zúñiga Rodríguez, L. (2015). Culpables, millonarios e impunes: el difícil tratamiento del derecho penal del delito de cuello blanco. Revista IUS, 9(35), 37-57. Disponible en: http://goo.gl/UoUGeg 